\title{
Readmissions after Pancreatic Surgery in Patients with Pancreatic Cancer: Does Hospital Variation Exist for Quality Measurement?
}

\author{
Ching-Yu Wang Joshua Brown \\ Center for Drug Evaluation \& Safety, Department of Pharmaceutical Outcomes and Policy, College of Pharmacy, \\ University of Florida, Gainesville, FL, USA
}

Keywords

Readmission rate $\cdot$ Administrative data $\cdot$ Pancreatic surgery $\cdot$ Quality of care

\begin{abstract}
Background: The appropriateness of using readmission rate after pancreatic surgery among pancreatic cancer patients as a quality metric to evaluate hospital performance has been widely discussed in the literature. Objectives: The present study reported readmission rate using Nationwide Readmissions Database (NRD), examined the reasons and risk factors for readmissions, and evaluated the appropriateness of using it as a quality metric. Method: We analyzed 3,619 patient discharge records in 2014. The outcome of interest was all-cause 30-day readmission. Reasons for readmission were grouped into clinical associated categories. Hierarchical regression model was used for examining the relationship between risk factors and readmission. Results: The 30-day readmission rate was $20.95 \%$. The most common reason for readmission was surgery-related complication. In descriptive analyses, age, certain comorbidities, number of chronic conditions, mortality risk, severity of illness, living at large metropolitan area, resident of the state where patients received initial care, postoperative complication, length of stay, discharge location, and receiving care at the hospitals in large metropolitan
\end{abstract}

area were predictive of readmission. In multivariable analysis, age, depression, peripheral vascular disorder, mortality risk, and discharge location were independently associated with readmission. The intraclass correlation coefficient was 0.41 for hierarchical regression model. Conclusions: Readmission after pancreatic surgery remains an important issue. Our study found the majority of variation in readmissions is accounted for by patient factors whereas there was little between hospital variation. This finding does not support the use of readmission rate after pancreatic surgery as a quality metric.

(c) 2019 S. Karger AG, Basel

\section{Introduction}

Initiatives to lower escalating healthcare costs over the last decade have included efforts to reduce unplanned readmissions in selected conditions. In 2012, the Centers for Medicare and Medicaid Services (CMS) started imposing financial penalties, i.e., reduced reimbursements, on hospitals with excess 30-day readmissions after certain treatment and disease conditions [1]. Currently, six medical conditions are included in the readmission reduction program including acute myocardial infarction, heart failure, pneumonia, chronic ob- 
structive pulmonary disease, coronary artery bypass graft surgeries, and elective primary total hip and/or total knee arthroplasty [1]. Readmission rates are also considered as a means to measure healthcare quality and have been utilized to measure individual hospital performance.

Although the list of conditions has not yet included any general surgery, surgeons and health service researchers have taken a proactive step to understand the reasons and risk factors for readmission after certain types of surgeries as a means to measure quality and reduce costs. Gastrointestinal operations have been discussed in the literature due to their higher readmission rate as compared to non-gastrointestinal operations [2]. As an example, one prior study found that readmissions after colon resection predicted 1-year mortality for patients with colon cancer. Based on those findings, the authors concluded that readmission may be an important metric to evaluate the quality of colon cancer surgery as it is closely tied to patient outcomes [3]. This conclusion led to further interest in other gastrointestinal surgeries and the potential of readmission rates as a quality indicator.

Pancreatic surgery might be an ideal target due to high readmission rates and frequent postoperative morbidity. Pancreatic surgery is the standard of care and only curative treatment for early-stage pancreatic cancer with modern advancements in perioperative management and surgical techniques during the past few decades [4]. However, high readmission rates still cast a shadow over its usefulness; thus, evaluations to determine the drivers of these rates, such as hospital quality, are needed.

Several studies have investigated this topic, but variations existed in approach. Studies differed in the time frame used for readmission, the definition of risk factors, surgery types, and the data source [5]. Not surprisingly, the results of these studies were also different, with reported readmission rates ranging from $6 \%$ to $59 \%[6,7]$. Discrepancy also existed in the reasons and the risk factors for readmission [5]. While some studies found patient-level risk factors contribute most to the readmissions [8], others found hospital surgery volume had a significant impact not only on the readmissions but also on other postoperative outcomes [9]. Thus, a better understanding of the risk factors and reasons for readmission is essential to evaluate the contribution of hospitals and potential interventions to reduce readmissions. The present study aimed to (1) estimate the readmission rate after pancreatic surgery among a national cohort of pancreatic cancer patients, (2) report the reasons for readmissions, (3) identify the risk factors associated with the readmissions, and (4) evaluate the contribution of individual hospitals on readmission rates.

\section{Materials and Methods}

A retrospective cohort study was performed using the Healthcare Cost and Utilization Project's (HCUP) Nationwide Readmissions Database (NRD) in 2014. The NRD is an all-payer database, including uninsured, of hospital inpatient stays and discharges from 22 states that accounts for $49.3 \%$ of all United States hospitalizations [10]. The data are representative of the national population, are de-identified, and were considered exempt by the University of Florida Institutional Review Board.

Index hospitalizations were selected based on the following inclusion criteria: (1) adult patients aged $\geq 18$ years, (2) a primary diagnosis of pancreatic cancer based on International Classification of Diseases, Ninth Revision, Clinical Modification (ICD-9CM) diagnosis codes (ICD-9-CM 157, 157.0-157.9), and (3) any pancreatic surgery based on ICD-9-CM procedural codes (52.5, $52.51-52.53,52.59,52.6,52.7)$. We excluded records with the discharge date in December to allow assessment of 30-day readmissions. We further excluded records indicating death during hospitalizations to assess only patients at risk of readmission after discharge. For patients with more than two discharge records that met the inclusion and exclusion criteria, only the first records were counted as index hospitalizations. The flow chart for sample selection is shown in online supplementary Figure 1 (for all online suppl. material, see www.karger.com/doi/10.1159/000502894).

Potential risk factors can be categorized into two levels: patient level and hospital level. Patient-level risk factors included age, sex, comorbidities, the number of chronic conditions, risk of mortality, severity of illness, household income, patient location, state residence and insurance status, index hospitalization length of stay, procedure type, common postoperative complications, and discharge location (e.g., home). Comorbidity measures were identified by the AHRQ comorbidity coding that identifies preexisting medical conditions and were calculated within the NRD data. Number of chronic conditions contains the count of unique chronic diagnoses reported on the discharge record and was also calculated within NRD data. Risk of mortality and severity of illness refer to the likelihood of dying and the extent of physiologic decompensation or organ system loss of function. These variables were also calculated by AHRQ and are included in the NRD with classifications of minor, moderate, major, and extreme assigned to each discharge record. Surgery procedure type was categorized as pancreaticoduodenectomy, distal pancreatectomy, and/or total pancreatectomy. Postoperative complication during the index hospitalization was based on relevant ICD-9-CM diagnosis codes (online supplementary Table 1). Hospital-level risk factors included hospital bed count, hospital cancer discharge volume, hospital pancreatic cancer discharge volume, hospital pancreatic surgery volume, hospital location, and hospital ownership (e.g., nonprofit). The effect of hospital size on readmission was inconsistent in the existing literature; thus, we aimed to understand whether hospital size has an impact on readmission by using four different measures representing hospital size [5]. Hospital bed count was calculated within NRD data. Hospitals were classified as small, medium, and large based on region, location, teaching status of the hospitals, and their number of beds. Hospital cancer/pancreatic cancer discharge volume were based on the number of cancer/pancreatic cancer patients for each hospital discharge in 2014; hospital pancreatic surgery volume was based on the number of pancreatic surgeries per each hospital performed in 2014. Hospitals were grouped into quartiles and classified as very low, low, medium, and high volume for each category.

The outcome of interest was all-cause 30-day readmission, defined as any hospital admission that occurred within 30 days of discharge from the index hospitalization date. Reasons for read- 
mission were based on the principal diagnosis code of the readmission record. These codes were then grouped into clinically associated categories (online supplementary Table 2) based on existing literature.

Risk factors were summarized by mean and standard deviation for continuous variables and frequency and percentage for categorical variable and compared between readmitted patients and non-readmitted patients using chi-squared, Fisher's exact, and independent $t$ tests. Cells with fewer than 11 discharge records were relabeled as NR in compliance with HCUP data use agreement. We conducted a subgroup analysis on patients who were residents of the state where he or she received hospital care to test the robustness of study results based on residence status.

All tests were 2 -sided, and a $p$ value $<0.05$ was considered statistically significant. Hierarchical generalized linear model was used due to the hierarchical structure between patient and hospital. The intraclass correlation coefficient (ICC) estimates how much variation in the readmission exists between hospitals. Risk factors were included in the final model if they differed significantly between readmission and non-readmission cohort, or if they were found independently associated with readmission in previous studies. All statistical analyses were performed using SAS software (version 9.4; SAS Institute Inc., Cary, NC, USA).

\section{Results}

A total of 3,619 index hospitalizations (patients) were identified during the study period; 758 were readmitted within 30 days after discharge (21\%). The median time to readmission for readmitted patients was 8 days (interquartile range, 4-16 days). The distributions of time to readmission are shown in Figure 1 and reasons for these readmissions are summarized in Table 1 . The mean age at operation was 66 years and there was an average of 6.2 chronic conditions. Most patients resided in large metropolitan areas and utilized Medicare or private insurance. The most frequent operation performed was pancreaticoduodenectomy. Postoperative complications occurred in $28.4 \%$ of the patients. Patient-level risk factors of the study population, stratified by readmission, are shown in Table 2 .

Pancreatic surgeries $(n=3,619)$ were performed at 405 different hospitals and pancreatic surgery volume in 2014 ranged from 1 to 193, with a median of 4 surgeries. Patients generally underwent pancreatic surgeries at larger hospitals $(78.5 \%)$, with high cancer discharge volumes (96.2\%), high pancreatic cancer discharge volumes (94.3\%), and high pancreatic surgery volumes (72.8\%). Hospital-level risk factors are shown in Table 3. More patients in the readmitted cohort were admitted to a hospital in large metropolitan areas $(80.1 \%$ vs. $75.8 \%, p$ value $=0.0302)$. Other hospital attributes did not significantly differ.

The independent predictors for 30-day readmission included age, depression, peripheral vascular disorder, risk of mortality, and discharge location (Table 4). Compared to ages 18-60, patients aged 61-70 years had higher odds of experiencing readmission $(\mathrm{OR}=1.25, \mathrm{CI}=$
Table 1. Reasons for readmission among patients undergoing pancreatic surgery

\begin{tabular}{ll}
\hline Reasons for readmission & $\begin{array}{l}\text { Frequency, } \\
\%\end{array}$ \\
\hline Surgery-related complication & $264(34.8)$ \\
Infection & $117(15.4)$ \\
Gastrointestinal dysfunction & $92(12.1)$ \\
Failure to thrive & $46(6.1)$ \\
Progression of disease & $25(3.3)$ \\
Venous thromboembolism/pulmonary embolism & $23(3)$ \\
Pain & $22(2.9)$ \\
Diabetes & $22(2.9)$ \\
Cardiac & $19(2.5)$ \\
Pancreatic-biliary disorder & $15(2)$ \\
Anemia and other hematologic disorder & $10(1.3)$ \\
Other & $97(12.7)$ \\
\hline
\end{tabular}

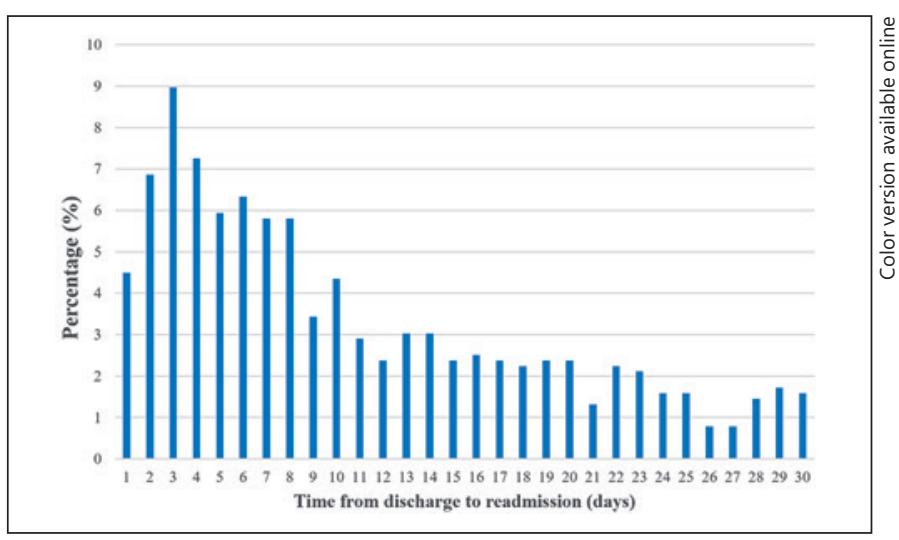

Fig. 1. The distributions of time from discharge to readmission.

$1.00-1.55)$. Patients with depression and peripheral vascular disorder were more likely to be readmitted $(\mathrm{OR}=$ $1.41, \mathrm{CI}=1.09-1.84$; OR $=1.46, \mathrm{CI}=1.06-2.01)$. Compared to those discharged home, patients who were discharged with home health care or to other facilities had higher odds of readmission ( $\mathrm{OR}=1.71, \mathrm{CI}=1.30-2.25$; $\mathrm{OR}=1.3, \mathrm{CI}=1.08-1.57)$. The ICC was $0.41 \%$, which indicates the proportion of variability in the readmission rate accounted for by the hospital. The c-statistic of the model was 0.63 , showing low discriminatory ability between readmitted and non-readmitted patients. The subgroup analysis was consistent with the main analysis (data not shown).

\section{Discussion/Conclusion}

Several patient-level risk factors were found to be associated with readmission including the presence of comorbidities, postoperative complication, patient loca- 
Table 2. Comparison of patient-level risk factors based on readmission status in patients with an index pancreatic surgery admission

\begin{tabular}{|c|c|c|c|c|}
\hline & $\begin{array}{l}\text { Total cohort } \\
(n=3,619)\end{array}$ & $\begin{array}{l}\text { Readmission } \\
(n=758)\end{array}$ & $\begin{array}{l}\text { No readmission } \\
(n=2,861)\end{array}$ & $p$ value \\
\hline Age, years (SD) & $66(11.2)$ & $66.6(10.6)$ & $65.8(11.3)$ & 0.0645 \\
\hline Age & & & & 0.0141 \\
\hline $18-60$ years & $1,055(29.2)$ & $187(24.7)$ & $868(30.3)$ & \\
\hline $61-70$ years & $1,240(34.3)$ & $285(37.6)$ & $955(33.4)$ & \\
\hline $71-80$ years & $1,030(28.5)$ & $227(30.0)$ & $803(28.1)$ & \\
\hline 81-90 years & $294(8.1)$ & $59(7.8)$ & $235(8.2)$ & \\
\hline Sex, $n(\%)$ & & & & 0.4117 \\
\hline Male & $1,867(51.6)$ & $381(51.9)$ & $1,486(50.3)$ & \\
\hline Female & $1,752(48.4)$ & $377(48.1)$ & $1,375(49.7)$ & \\
\hline \multicolumn{5}{|l|}{ Comorbidities, $n(\%)$} \\
\hline Alcohol abuse & $115(3.2)$ & $27(3.6)$ & $88(3.1)$ & 0.4975 \\
\hline Anemia & $600(16.6)$ & $137(18.1)$ & $463(16.2)$ & 0.2133 \\
\hline Congestive heart failure & $119(3.3)$ & $35(4.6)$ & $84(2.9)$ & 0.0210 \\
\hline Coagulopathy & $212(5.9)$ & $51(6.7)$ & $161(5.6)$ & 0.2512 \\
\hline Depression & $345(9.5)$ & $97(12.8)$ & $248(8.7)$ & 0.0006 \\
\hline Diabetes & $1,298(35.9)$ & $268(35.4)$ & $1,030(36)$ & 0.7419 \\
\hline Pulmonary disease & $585(16.16)$ & $152(20.1)$ & $433(15.1)$ & 0.0011 \\
\hline Drug abuse & $49(1.4)$ & $12(1.6)$ & $37(1.3)$ & 0.5392 \\
\hline Hypertension & $2,160(59.7)$ & $467(61.6)$ & $1,693(59.2)$ & 0.2244 \\
\hline Hypothyroidism & $440(12.2)$ & $95(12.5)$ & $345(12.1)$ & 0.7224 \\
\hline Liver disease & $182(5)$ & $49(6.5)$ & $133(4.7)$ & 0.0420 \\
\hline Fluid and electrolyte disorders & $1,135(31.4)$ & $279(36.8)$ & $856(29.9)$ & 0.0003 \\
\hline Metastatic cancer & $1,286(35.5)$ & $274(36.15)$ & $1,012(35.37)$ & 0.6916 \\
\hline Obesity & $403(11.1)$ & $87(11.5)$ & $316(11.1)$ & 0.7365 \\
\hline Renal failure & $201(5.6)$ & $49(6.5)$ & $152(5.3)$ & 0.2184 \\
\hline Peptic ulcer disease & NR & NR & NR & 0.9586 \\
\hline Weight loss & $672(18.6)$ & $151(19.9)$ & $521(18.2)$ & 0.2816 \\
\hline Valvular heart disease & $152(4.2)$ & $32(4.2)$ & $120(4.2)$ & 0.9734 \\
\hline Peripheral vascular disorder & $207(5.7)$ & $63(8.3)$ & $144(5)$ & 0.0005 \\
\hline Rheumatoid arthritis/collagen vascular disease & $81(2.2)$ & $16(2.1)$ & $65(2.3)$ & 0.7898 \\
\hline Neurological disorders & $127(3.5)$ & $31(4.1)$ & $96(3.4)$ & 0.3287 \\
\hline Psychoses & $82(2.3)$ & $19(2.5)$ & $63(2.2)$ & 0.6164 \\
\hline Number of chronic conditions & $6.2(3.0)$ & $6.7(3.1)$ & $6.1(2.9)$ & $<0.0001$ \\
\hline \multicolumn{4}{|l|}{ Number of chronic conditions } & $<0.0001$ \\
\hline$\leq 9$ & $3,122(86.3)$ & $621(81.93)$ & $2,501(87.4)$ & \\
\hline$>9$ & $497(13.7)$ & $137(18.07)$ & $360(12.6)$ & \\
\hline \multicolumn{4}{|l|}{ Mortality risk } & $<0.0001$ \\
\hline Minor & $1,038(26.7)$ & $180(23.8)$ & $858(30)$ & \\
\hline Moderate & $1,375(38)$ & $265(35)$ & $1,110(39)$ & \\
\hline Major & $852(23.5)$ & $218(28.8)$ & $634(22.2)$ & \\
\hline Extreme & $354(9.8)$ & $95(12.5)$ & $259(9.1)$ & \\
\hline \multicolumn{4}{|l|}{ Severity of illness } & $<0.0001$ \\
\hline Minor & $164(4.5)$ & $27(3.6)$ & $137(4.8)$ & \\
\hline Moderate & $665(18.4)$ & $143(18.9)$ & $522(18.3)$ & \\
\hline Major & $2,345(64.8)$ & $459(60.55)$ & $1,886(65.9)$ & \\
\hline Extreme & $445(12.3)$ & $129(17)$ & $316(11.1)$ & \\
\hline \multicolumn{4}{|l|}{ Median household income, $n(\%)$} & 0.6532 \\
\hline $0-25$ th & $725(20.2)$ & $156(20.9)$ & $567(20.1)$ & \\
\hline $26-50$ th & $889(24.9)$ & $177(23.7)$ & $712(25.2)$ & \\
\hline $51-75$ th & $851(23.8)$ & $172(23)$ & $679(24)$ & \\
\hline 76-100th & $110(31.1)$ & $243(32.5)$ & $867(30.7)$ & \\
\hline \multicolumn{4}{|l|}{ Patient location, $n(\%)$} & 0.0351 \\
\hline Large metropolitan area & $2,122(58.9)$ & 475 (62.9) & $1,647(57.8)$ & \\
\hline Small metropolitan areas & $1,303(36.1)$ & $248(32.9)$ & $1,055(37)$ & \\
\hline Other areas & $181(5)$ & $32(4.2)$ & $149(5.2)$ & \\
\hline \multicolumn{4}{|l|}{ Resident status, $n(\%)$} & 0.0491 \\
\hline Nonresident & 479 (13.2) & 84 (11.9) & 395 (13.8) & \\
\hline Resident & $3,140(86.8)$ & $674(88.9)$ & $2,466(86.2)$ & \\
\hline
\end{tabular}


Table 2 (continued)

\begin{tabular}{|c|c|c|c|c|}
\hline & $\begin{array}{l}\text { Total cohort } \\
(n=3,619)\end{array}$ & $\begin{array}{l}\text { Readmission } \\
(n=758)\end{array}$ & $\begin{array}{l}\text { No readmission } \\
(n=2,861)\end{array}$ & $p$ value \\
\hline Insurance status, $n(\%)$ & & & & 0.1881 \\
\hline Medicare & $2,057(57)$ & $456(60.2)$ & $4,601(56.1)$ & \\
\hline Medicaid & $265(7.3)$ & $50(6.6)$ & $215(7.5)$ & \\
\hline Private including $\mathrm{HMO}$ & $1,174(32.5)$ & $223(29.5)$ & $951(33.3)$ & \\
\hline Self-pay & $62(1.7)$ & $14(1.9)$ & $48(1.7)$ & \\
\hline Others (including no charges) & $54(1.5)$ & $14(1.9)$ & $40(1.4)$ & \\
\hline Mean length of stay, days (SD) & $11.2(8.9)$ & $12.40(10.2)$ & $10.93(8.5)$ & 0.0003 \\
\hline Length of stay & & & & 0.0008 \\
\hline$\leq 6$ days & $992(27.4)$ & $171(22.6)$ & $821(28.7)$ & \\
\hline$>6$ days & $2,627(72.6)$ & $587(77.4)$ & $2,040(71.3)$ & \\
\hline Pancreaticoduodenectomy & $2,469(68.2)$ & $516(68.1)$ & $1,953(68.3)$ & 0.9209 \\
\hline Distal pancreatectomy & $828(22.9)$ & $178(23.5)$ & $650(22.7)$ & 0.6564 \\
\hline Total pancreatectomy & $160(4.4)$ & $33(4.4)$ & $127(4.4)$ & 0.9190 \\
\hline Common postoperative complications & & & & 0.0128 \\
\hline Yes & $1,029(28.43)$ & $243(32.06)$ & $786(27.47)$ & \\
\hline No & $2,590(71.57)$ & $515(67.94)$ & $2,075(72.53)$ & \\
\hline Discharge location & & & & $<0.0001$ \\
\hline Home & $1,921(53.1)$ & $335(44.2)$ & $1,586(55.4)$ & \\
\hline Home healthcare & $1,253(34.6)$ & $290(38.3)$ & $963(33.7)$ & \\
\hline Other facilities & $443(12.2)$ & $132(17.4)$ & $311(10.9)$ & \\
\hline AMA & NR & NR & NR & \\
\hline
\end{tabular}

Table 3. Comparison of hospital-level risk factors based on readmission status

\begin{tabular}{|c|c|c|c|c|}
\hline & $\begin{array}{l}\text { Total cohort } \\
(n=3,619)\end{array}$ & $\begin{array}{l}\text { Readmission } \\
(n=758)\end{array}$ & $\begin{array}{l}\text { No readmission } \\
(n=2,861)\end{array}$ & $p$ value \\
\hline Hospital size (bed count), $n(\%)$ & & & & 0.5238 \\
\hline Small & $238(6.6)$ & $43(5.7)$ & $195(6.8)$ & \\
\hline Medium & $540(14.9)$ & $113(14.9)$ & $427(14.9)$ & \\
\hline Large & $2,841(78.5)$ & $602(79.4)$ & $2,239(78.3)$ & \\
\hline Hospital size (cancer discharge volume), $n(\%)$ & & & & 0.4557 \\
\hline Very low & $0(0)$ & $0(0)$ & $0(0)$ & \\
\hline Low & $\mathrm{NR}$ & $\mathrm{NR}$ & NR & \\
\hline Medium & $128(3.5)$ & $99(3.5)$ & $29(3.8)$ & \\
\hline High & $3,483(96.2)$ & $2,757(96.36)$ & $726(95.8)$ & \\
\hline Hospital size (pancreatic cancer discharge volume), $n(\%)$ & & & & 0.4709 \\
\hline Very low & $0(0)$ & $0(0)$ & $0(0)$ & \\
\hline Low & $30(0.8)$ & NR & $21(0.7)$ & \\
\hline Medium & $175(4.8)$ & $37(4.9)$ & $138(4.8)$ & \\
\hline High & $3,414(94.3)$ & $712(93.9)$ & $2,702(94.4)$ & \\
\hline Hospital size (pancreatic surgery volume), $n(\%)$ & & & & 0.1014 \\
\hline Very low & $105(2.9)$ & $24(3.2)$ & $81(2.8)$ & \\
\hline Low & $349(9.6)$ & $58(7.65)$ & $291(10.2)$ & \\
\hline Medium & $530(14.6)$ & $102(13.5)$ & $428(15)$ & \\
\hline High & $2,635(72.8)$ & $574(75.7)$ & $2,061(72)$ & \\
\hline Ownership, $n(\%)$ & & & & 0.8037 \\
\hline Government, nonfederal & $436(12.1)$ & $89(11.7)$ & $347(12.1)$ & \\
\hline Private, nonprofit & $2,957(81.7)$ & $618(81.5)$ & $2,339(81.8)$ & \\
\hline Private, investor-owned & $226(6.2)$ & $51(6.7)$ & $175(6.1)$ & \\
\hline Hospital location, $n(\%)$ & & & & 0.0302 \\
\hline Large metropolitan area & $2,776(76.7)$ & $607(80.1)$ & $2,169(75.8)$ & \\
\hline Small metropolitan area & $833(23)$ & $148(19.5)$ & $685(23.9)$ & \\
\hline Other & NR & NR & NR & \\
\hline
\end{tabular}


Table 4. Multivariable hierarchical regression model of 30-day readmission in 3,619 patients after pancreatic surgery

\begin{tabular}{|c|c|c|}
\hline & Adj. OR & CI \\
\hline \multicolumn{3}{|l|}{ Age } \\
\hline $18-60$ years & Ref. & Ref. \\
\hline $61-70$ years & 1.25 & $1.00-1.55^{\star}$ \\
\hline $71-80$ years & 1.07 & $0.84-1.36$ \\
\hline $81-90$ years & 0.80 & $0.56-1.15$ \\
\hline \multicolumn{3}{|l|}{ Sex } \\
\hline Male & Ref. & Ref. \\
\hline Female & 1.03 & $0.87-1.22$ \\
\hline \multicolumn{3}{|l|}{ Comorbidities } \\
\hline Hypertension & 1.02 & $0.86-1.23$ \\
\hline Congestive heart failure & 1.21 & $0.79-1.86$ \\
\hline Depression & 1.41 & $1.09-1.84^{*}$ \\
\hline Pulmonary disease & 1.16 & $0.93-1.44$ \\
\hline Metastatic cancer & 0.97 & $0.80-1.18$ \\
\hline Liver disease & 1.28 & $0.90-1.81$ \\
\hline Fluid and electrolyte disorders & 1.16 & $0.97-1.40$ \\
\hline Peripheral vascular disorder & 1.46 & $1.06-2.01^{*}$ \\
\hline Diabetes & 0.86 & $0.72-1.03$ \\
\hline \multicolumn{3}{|l|}{ Number of comorbidities } \\
\hline$\leq 9$ & Ref. & Ref. \\
\hline$>9$ & 1.11 & $0.86-1.42$ \\
\hline \multicolumn{3}{|l|}{ Mortality risk } \\
\hline Minor & Ref. & Ref. \\
\hline Moderate & 1.07 & $0.84-1.37$ \\
\hline Major & 1.37 & $1.03-1.81^{*}$ \\
\hline Extreme & 1.44 & $0.98-2.12$ \\
\hline \multicolumn{3}{|l|}{ Patient location } \\
\hline Large metropolitan area & Ref. & Ref. \\
\hline Small metropolitan areas & 0.88 & $0.70-1.11$ \\
\hline Other areas & 0.76 & $0.50-1.18$ \\
\hline \multicolumn{3}{|l|}{ Resident status } \\
\hline Nonresident & Ref. & Ref. \\
\hline Resident & 1.27 & $0.96-1.67$ \\
\hline \multicolumn{3}{|l|}{ Length of stay } \\
\hline$\leq 6$ days & Ref. & Ref. \\
\hline$>6$ days & 1.21 & $0.97-1.51$ \\
\hline Pancreaticoduodenectomy & 0.98 & $0.67-1.45$ \\
\hline Distal pancreatectomy & 1.18 & $0.79-1.78$ \\
\hline Total pancreatectomy & 0.79 & $0.45-1.39$ \\
\hline \multicolumn{3}{|l|}{ Common postoperative complications } \\
\hline No & Ref. & Ref. \\
\hline Yes & 0.98 & $0.80-1.20$ \\
\hline \multicolumn{3}{|l|}{ Discharge location } \\
\hline Home & Ref. & Ref. \\
\hline Home healthcare & 1.71 & $1.30-2.25^{*}$ \\
\hline Other facilities & 1.30 & $1.08-1.57^{*}$ \\
\hline AMA & 5.61 & $0.31-100.59$ \\
\hline \multicolumn{3}{|l|}{ Hospital location } \\
\hline Large metropolitan area & Ref. & Ref. \\
\hline Small metropolitan area & 0.87 & $0.66-1.15$ \\
\hline Other & 2.27 & $0.55-9.34$ \\
\hline \multicolumn{3}{|c|}{ Hospital size (pancreatic surgery volume) } \\
\hline Very low & Ref. & Ref. \\
\hline Low & 0.68 & $0.39-1.18$ \\
\hline Medium & 0.86 & $0.51-1.44$ \\
\hline High & 1.07 & $0.66-1.74$ \\
\hline C-statistic & 0.63 & \\
\hline ICC, \% & 0.41 & \\
\hline
\end{tabular}

* Statistically significant at $\alpha=0.05$. ICC, intraclass correlation coefficient; Adj. OR, odds ratio adjusted for all other covariates in the model; CI, $95 \%$ confidence interval. tion, and insurance status in descriptive analysis. It was worth noting that length of stay has a positive association with readmission before adjustment but was not an independent predictor after accounting for other covariates. While there was a possibility that early discharge (shorter length of stay) precipitated readmission, it was not the case in this patient population. Length of stay possibly represented the severity of condition and itself was not predictive of readmission.

In contrast to patient-level risk factors, hospital-level risk factors appeared to have a minimal effect on risk for readmission. None of the hospital-level risk factors were predictors for readmission in this study, which was further confirmed by the low ICC in the risk model. While previous studies found inconsistent associations between hospital surgery volume and readmission $[8,11]$, our analysis did not show a volume-readmission relationship. This might be due to the large number of low-volume hospitals in our data. Only one pancreatic surgery was performed in $25 \%$ of the hospitals. This volume-readmission relationship might exist among more experienced hospitals and future studies are needed.

This study has two important implications. From the perspective of policymakers, the low ICC implies that there is insignificant variation in quality among hospitals in terms of readmission after pancreatic surgery. This calls into question the appropriateness of using readmission rate after pancreatic surgery as a quality measure. Prior studies have concluded similar assessments driven by the unpredictable nature of readmissions [12], the lack of evidence-based guidelines for the management of perioperative complication [13], and the high variation in readmission among patients but not among hospitals [8]. From the clinical standpoint, certain populations are prone to readmission including those with certain preexisting comorbidities and those aged over 60 . Healthcare providers utilizing this information can pay more attention to patients that are at higher risk of readmission and ultimately improve the quality of care.

The strength of the study was that we used a national representative database that covers all age groups and all payers; thus, our result can be generalized to the entire United States. Our study is unique because we overcame the inability of single institutional database to track readmissions to non-index hospitals. We also utilized a multilevel model to account for the hospital clustering effect and measure the contribution of hospitals to the risk of readmission. There are also several limitations. First, NRD is unable to track readmissions outside of a state where index hospitalization occurred. Hence, some readmissions may not be included in our analysis if the patients were readmitted in a different state. However, we tested the impact of these omitted readmissions by conducting a subgroup analysis on state residents who were 
less likely to cross state borders to be readmitted, which was consistent with the main analysis and proved the robustness of the study result. Since NRD is derived from billing information, it lacks detailed clinical information, e.g., lab values and biometric data. Previous studies found vessel resection [7] and cancer stage [6] were independently associated with readmission; however, we were unable to account for these variables. Lastly, NRD does not track death outside of the hospital. However, the impact of this limitation was likely to be minimal given the median survival after pancreaticoduodenectomy is between 10 and 20 months [14-20].

In conclusion, readmission remains a problem for pancreatic cancer patients who underwent pancreatic surgeries with 1 in 5 patients readmitted to the hospital within 30 days after discharge, most often for surgeryrelated complications. Our finding suggests that variations in readmission are attributed to patient-level risk factors or additional unmeasured variables with hospitallevel risk factors having only a minor role in readmission. This finding does not support the use of readmission rate after pancreatic surgery as a quality metric. Future studies on more experienced hospitals, e.g., by surgery volume, are necessary to make a sound recommendation on post- surgical readmission quality metrics. Meanwhile, more direct indicators of pancreatic surgery quality, e.g., R0 resection rate, postoperative complication rates, etc., should be further emphasized.

\section{Statement of Ethics}

The authors have no ethical conflicts.

\section{Disclosure Statement}

The authors have no conflicts of interest to disclose.

\section{Funding Sources}

No funding was provided for this research.

\section{Author Contributions}

C.-Y.W. performed the analysis and wrote the manuscript. J.B. edited the manuscript and provided intellectual input.

\section{References}

1 Centers for Medicare and Medicaid Services (CMS). Readmissions Reduction Program (HRRP). https://www.cms.gov/medicare/ medicare-fee-for-service-payment/acuteinpatientpps/readmissions-reduction-program.html. Accessed October 15, 2018.

2 Martin RC, Brown R, Puffer L, Block S, Callender G, Quillo A, et al. Readmission rates after abdominal surgery: the role of surgeon, primary caregiver, home health, and subacute rehab. Ann Surg. 2011 Oct;254(4): 591-7.

3 Greenblatt DY, Weber SM, O'Connor ES, LoConte NK, Liou JI, Smith MA. Readmission after colectomy for cancer predicts one-year mortality. Ann Surg. 2010 Apr;251(4):65969.

4 National Comprehensive Cancer Network (NCCN). Pancreatic Adenocarcinoma (version 2.2018). 2018; https://www.nccn.org/ professionals/physician_gls/pdf/pancreatic. pdf. Accessed October 15, 2018.

5 Fisher AV, Fernandes-Taylor S, CampbellFlohr SA, Clarkson SJ, Winslow ER, Abbott DE, et al. 30-day Readmission After Pancreatic Resection: A Systematic Review of the Literature and Meta-analysis. Ann Surg. 2017 Aug;266(2):242-50.

6 Yermilov I, Bentrem D, Sekeris E, Jain S, Maggard MA, Ko CY, et al. Readmissions following pancreaticoduodenectomy for pancreas cancer: a population-based appraisal. Ann Surg Oncol. 2009 Mar;16(3): 554-61.
7 Ahmad SA, Edwards MJ, Sutton JM, Grewal SS, Hanseman DJ, Maithel SK, et al. Factors influencing readmission after pancreaticoduodenectomy: a multi-institutional study of 1302 patients. Ann Surg. 2012 Sep;256(3): 529-37.

8 Hyder O, Dodson RM, Nathan H, Schneider $\mathrm{EB}$, Weiss MJ, Cameron JL, et al. Influence of patient, physician, and hospital factors on 30day readmission following pancreatoduodenectomy in the United States. JAMA Surg. 2013 Dec;148(12):1095-102.

9 Krautz C, Denz A, Weber GF, Grützmann R. Influence of Hospital Volume Effects and Minimum Caseload Requirements on Quality of Care in Pancreatic Surgery in Germany. Visc Med. 2017 May;33(2):131-4.

10 Healthcare Cost and Utilization Project (HCUP). Introduction to the HCUP nationwide readmission database. NRD; 2014.

11 Sutton JM, Wilson GC, Wima K, Hoehn RS, Cutler Quillin R 3rd, Hanseman DJ, et al. Readmission After Pancreaticoduodenectomy: The Influence of the Volume Effect Beyond Mortality. Ann Surg Oncol. 2015 Nov;22(12):3785-92.

12 Kastenberg ZJ, Morton JM, Visser BC, Norton JA, Poultsides GA. Hospital readmission after a pancreaticoduodenectomy: an emerging quality metric? HPB (Oxford). 2013 Feb; 15(2):142-8.

13 Gawlas I, Sethi M, Winner M, Epelboym I, Lee JL, Schrope BA, et al. Readmission after pancreatic resection is not an appropriate measure of quality. Ann Surg Oncol. 2013 Jun;20(6):1781-7.
14 Yeo CJ, Cameron JL, Sohn TA, Lillemoe KD, Pitt HA, Talamini MA, et al. Six hundred fifty consecutive pancreaticoduodenectomies in the 1990s: pathology, complications, and outcomes. Ann Surg. 1997 Sep;226(3):248-57.

15 Geer RJ, Brennan MF. Prognostic indicators for survival after resection of pancreatic adenocarcinoma. Am J Surg. 1993 Jan;165(1): 68-72; discussion 72-3.

16 Benassai G, Mastrorilli M, Quarto G, Cappiello A, Giani U, Mosella G. Survival after pancreaticoduodenectomy for ductal adenocarcinoma of the head of the pancreas. Chir Ital. 2000 May-Jun;52(3):263-70.

17 Millikan KW, Deziel DJ, Silverstein JC, Kanjo TM, Christein JD, Doolas A, et al. Prognostic factors associated with resectable adenocarcinoma of the head of the pancreas. Am Surg. 1999 Jul;65(7):618-23.

18 Bakkevold KE, Arnesjø B, Dahl O, Kambestad B. Adjuvant combination chemotherapy (AMF) following radical resection of carcinoma of the pancreas and papilla of Vater-results of a controlled, prospective, randomised multicentre study. Eur J Cancer. 1993;29A(5): 698-703.

19 Tsao JI, Rossi RL, Lowell JA. Pylorus-preserving pancreatoduodenectomy. Is it an adequate cancer operation. Arch Surg. 1994 Apr; 129(4):405-12.

20 Yeo CJ, Cameron JL, Lillemoe KD, Sitzmann JV, Hruban RH, Goodman SN, et al. Pancreaticoduodenectomy for cancer of the head of the pancreas. 201 patients. Ann Surg. 1995 Jun;221(6):721-31. 